\title{
NovaGenesis NameBindings: Avaliação de Virtualização em Escala
}

\author{
Victor Hugo D. D’Ávila ${ }^{1}$, Élcio C. do Rosário ${ }^{1}$, \\ José R. dos Santos ${ }^{1}$ e Antônio M. Alberti ${ }^{1}$ \\ ${ }^{1}$ ICT Lab - Instituto Nacional de Telecomunicações (INATEL) \\ Santa Rita do Sapucaí - MG - Brasil \\ victorhugo.davila@ifmg.edu.br, elcio.carlos@mtel.inatel.br, \\ joserodrigo@gec.inatel.br, alberti@inatel.br
}

\begin{abstract}
This article presents a scale evaluation of NovaGenesis content publishing and subscription services. This study complements previous works on this proposed Future Internet architecture and validates its services through experimentation in multiple scenarios.
\end{abstract}

Resumo. Este artigo apresenta uma avaliação em escala dos serviços de publicação e assinatura de conteúdos da NovaGenesis. O estudo complementa trabalhos prévios dessa proposta de arquitetura de Internet do Futuro e valida seus serviços através de experimentações em múltiplos cenários.

\section{Introdução}

A NovaGenesis (NG) [Alberti et al. 2017] é uma proposta de arquitetura de Internet do Futuro (IF) que possui desenho clean slate para nomeação, troca de dados, cache de rede e segurança da informação. O objetivo desse trabalho é realizar uma avaliação do mecanismo de resolução de nomes dessa arquitetura quando executado em múltiplos nós. A experimentação tornou-se importante porque a NG é estruturada por princípios diferentes da arquitetura atual de Internet e, por isso, a forma como seus elementos interagem deve ser testada em ambientes controlados e, se possível, comparada a outras propostas de IF.

A escolha do tema foi norteada pela necessidade de acompanhar as discussões sobre IF no cenário mundial. Essas discussões se baseiam na mudança de orientação do tráfego de dados que, em grande parte, deixa de ser orientado a hosts e passa ser orientado a conteúdos e informações [Conti et al. 2011]. Essa nova tendência, denominada Information Centric Network, vem sendo abordada nas propostas recentes de arquitetura de Internet e corroboram para suportar os novos requisitos de rede, as aplicações de Internet of Things (IoT), streaming massivo de vídeo, expansão do número de dispositivos móveis, mobilidade de usuários e segurança da informação.

Para alcançar o objetivo de testar em escala a NG foram utilizados softwares de virtualização e ambientes de experimentação de redes. Os resultados obtidos expõem as características da NovaGenesis e permitem acompanhar sua evolução. É esperado que a NG se destaque em alguns aspectos e seja eficiente em resolver nomes e localizar conteúdos armazenados na rede.

Esse artigo se organiza da seguinte forma: na Seção 2 são levantados os trabalhos relacionados ao tema. Na Seção 3 é definida a metodologia de trabalho para alcançar 
dados com relevância científica. Os dados são exibidos e analisados na Seção 4. Na Seção 5 estão as conclusões gerais e abordagens para trabalhos futuros.

\section{Trabalhos Relacionados}

Os trabalhos relacionados a esse tema são aqueles que tratam de experimentação de IF, redes de computadores, plataformas de experimentação e estudos sobre as tendências para a evolução da Internet. Os trabalhos que serão mencionados a seguir se encaixam em algum desses assuntos.

O CURLING [Chai et al. 2018] é uma proposta de arquitetura de IF que otimiza a transmissão de conteúdos na rede. Nela são utilizados os conceitos de Software-Defined Networking (SDN), com o desacoplamento da camada de dados e de controle, e é adicionada uma terceira camada, exclusiva para controle de conteúdos. Dessa forma, passa-se a ter uma camada especializada em lidar com a troca de conteúdos e caches intra-redes.

O Future Internet Brazilian Environment for Experimentation (FIBRE) [Salmito et al. ] é um testbed sediado no Brasil que proporciona a experimentação de arquiteturas de Internet do Futuro. O FIBRE foi base para os primeiros trabalhos da NG em ambientes de experimentação. Esse trabalho é uma continuidade e expansão dos testes realizados nesse testbed [Ferreira et al. 2017], em que foi definido um cenário com dois nós e avaliado o tempo de assinatura de NameBinds (NBs). Um NB é uma ligação entre nomes na forma $<X$, Nome(s) $>$, ou seja, um Nome $X$ é mapeado a um ou mais Nomes tanto em linguagem natural, quanto em nomes calculados via Função Hash. No trabalho citado foram avaliados o comportamento dos serviços da NG e do testbed, no trabalho corrente foram utilizados apenas servidores próprios.

\section{Metodologia}

Para realização desse trabalho foram elencados equipamentos e recursos que pudessem ser totalmente controlados e monitorados, fossem eles de hardware ou software. O principal equipamento utilizado foi um servidor com alta capacidade de processamento, descrito na Tabela 1. Como elementos de software foram utilizados: GNU/Linux, para o sistema operacional base do hypervisor e das VMs, Kernel-based Virtual Machine (KVM), como hypervisor, e a NovaGenesis, sendo executada dentro de cada VM e avaliada sob diferentes topologias.

Tabela 1. Configuração de hardware servidor de experimentação.

\begin{tabular}{l|l} 
Característica & Descrição \\
\hline Marca/Modelo & Dell PowerEdge T640 BCC \\
Processador & 2x Intel R Xeon TM Silver 4114 10 cores (2.20GHz, 13.75MB Cache, 9.6GT/s) \\
Memória & 256GB (8x32GB) RDIMM DDR4 2667 MT/s \\
Placa de rede & 2x 10GbE: Intel I350 (10GBase-T); 2x 1GbE: Intel I350 (1000Base-T) \\
Armazenamento & 2x SSD SATA 480GB 6 Gbps; 3x HDD SATA 4TB, 7.2K RPM \\
\hline
\end{tabular}

\subsection{KVM}

Para criação do ambiente virtual foi escolhido o hypervisor KVM dada a sua afinidade com plataformas de virtualização abertas e de código livre. Na Figura 1 é possível observar a organização dos elementos de software, desde o componente de hardware até o Sistema Operacional (SO) das VMs. Na camada mais baixa está o hardware do servidor. 


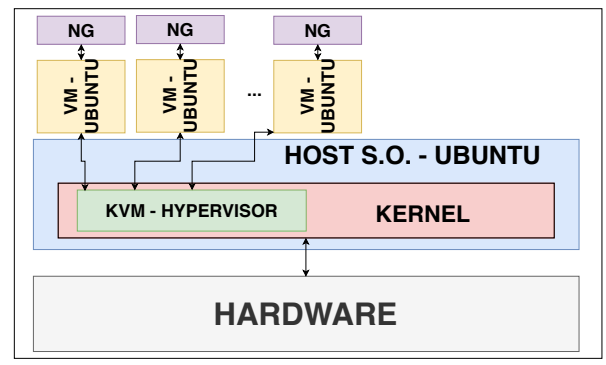

Figura 1. Topologia dos elementos de software da virtualização com o KVM.

Logo acima dele está o Kernel do SO do hospedeiro (host). O KVM é apresentado como parte do kernel. Esse é justamente seu diferencial em relação a outros hipervisores, ele é implementado diretamente no kernel do sistema hospedeiro para otimizar a comunicação de entrada/saída das VMs com o hardware. Na camada superior é possível observar o SO do host e as máquinas virtuais. As VMs são replicadas para atender a quantidade de nós necessários nos testes. A NG é executada em cada VM como um processo a nível de usuário.

\subsection{NovaGenesis}

$\mathrm{Na}$ NovaGenesis é utilizada uma abordagem para a troca de informações orientada a serviços. Isso facilita a expansão do número de dispositivos conectados em rede. Os três pilares da NovaGenesis são: resolução de nomes e cache de NBs e dados; ciclo de vida de serviços e conteúdos; e exposição de elementos de hardware como serviços. No primeiro pilar são propostas condições para mapear e armazenar conteúdos na rede. $\mathrm{O}$ segundo pilar trata todas as funcionalidades de rede como serviços e permite que eles comuniquem entre si através de Application Programming Interfaces (APIs). O último pilar transcende o mundo virtual e permite a representação dos elementos e dispositivos físicos como serviços de rede. Essa representação abre caminho para permitir o controle e compartilhamento de dispositivos de forma integrada [Alberti et al. 2018]. A NG é modular e seus componentes podem ser organizados em diferentes blocos, de acordo com a necessidade e/ou função que se deseja atribuir a um nó. Os serviços utilizados nesse trabalho foram:

- Proxy Gateway Controller Service (PGCS): É o serviço que inicia a exposição dos recursos e serviços de rede disponíveis em um domínio NovaGenesis. Também é o responsável pelo encapsulamento de mensagens NG sobre tecnologias de camada de enlace (e.g. Ethernet) e reconhecimento de outros nós NG em um domínio de rede.

- Publish/Subscribe Service (PSS): É o serviço responsável pelo recebimento das publicações e solicitações de assinaturas de NBs e conteúdos entre serviços e encaminhamento dos mesmos a uma instância de GIRS pré-descoberta visando balanceamento de carga.

- Generic Indirection Resolution Service (GIRS): Responsável por encontrar um Hash Table Service (HTS) apropriado para guardar os NBs e conteúdos associados.

- Hash Table Service (HTS): É o serviço responsável por armazenar NBs e conteúdos de forma distribuída e enviar as respostas de assinaturas ao serviço solicitante. 
- NBSimpleTestAPP: É a aplicação criada para gerar NBs aleatórios publicando-os e assinando-os, gerando métricas para avaliar o desempenho da arquitetura.

\subsection{NameBinds (NBs)}

NBs são associações relacionais entre duas entidades, sejam nomes, computadores, serviços, pessoas ou elementos que se desejem mapear. Para exemplificar essa associação é possível relembrar a relação que existe entre endereços IP e nomes de domínios na Internet. O site www.example.com pode ser associado ao endereço IP: 203.0.113.1 e vice-versa. Essa associação é considerada um NB. Nesse trabalho, os NBs são gerados por uma aplicação (NBSimpleTestAPP), que possui o único objetivo de publicar e assinar NBs via PSS.

\subsection{Topologias NovaGenesis}

A NG é executada como um conjunto dos serviços, de forma que a execução ou não de cada serviço caracteriza uma função diferente para o nó. Foram utilizados três tipos de nós nesses experimentos:

- Nó de controle (CORE): Executa o PGCS, GIRS e PSS. Tem função de receber publicações e assinaturas de NBs e encaminhar para o HTS correspondente.

- Nó de armazenamento (HTS): Executa o PGCS e HTS. Tem função característica de cache de NBs e conteúdos. É o responsável por armazenar as publicações e responder as solicitações de assinaturas de NBs do nó de controle.

- Nó de aplicação (APP): Executa o PGCS e NBSimpleTestAPP. Tem característica de aplicação. É o serviço que publica o NB para o CORE e os recebe via HTS. Contabiliza o tempo gasto na assinatura dos NBs pelo HTS.

Foram utilizadas quatro topologias na execução dos testes. O cenário mais simples é composto por duas VMs, uma executando a função de (CORE) e Armazenamento (HTS) da rede e outra executando a Aplicação (NBSimpleTestAPP), como mostra a Figura 2a. Com quatro VMs é possível inserir dois nós exclusivos de armazenamento na rede (HTS), conforme ilustrado na Figura 2b. Utilizando cinco VMs aumenta-se mais um nó de armazenamento (HTS), como mostra a Figura 2c. Com seis máquinas virtuais, uma VM executa o (CORE), outra a aplicação (APP) e quatro VMs são responsáveis pelo armazenamento (HTS) de conteúdos, tal qual mostrado na Figura 2d. Um dos focos desse experimento é justamente variar a quantidade de elementos de armazenamento (HTS) na rede e observar o seu desempenho. O número de publicações realizadas pela aplicação também varia de 1, 2, 4, 8, 16 e 32 milhões. O número de assinaturas realizadas após as publicações foi constante e definido em todos cenários como 360. Para cada cenário e nível de publicações foram realizados dez experimentos.

\section{Resultados e Discussões}

Os principais resultados foram compilados em gráficos de tempo médio de publicações e de assinatura de NBs de acordo com o cenário de teste. A Figura 3 exibe o tempo de publicação dos NBs utilizando de 1 a 4 HTSes. A Figura 4 exibe o tempo de assinatura dos NBs utilizando também de 1 a 4 HTSes.

Com o aumento no número de HTSes é possível observar um aumento no tempo de publicação e assinatura dos NBs. Esse resultado é consequência da troca de dados entre 
o nó principal (CORE) e os múltiplos nós HTS. Além disso, o GIRS passa a distribuir as solicitações de publicações e assinaturas, oriundas do (APP), para múltiplos HTSes. O

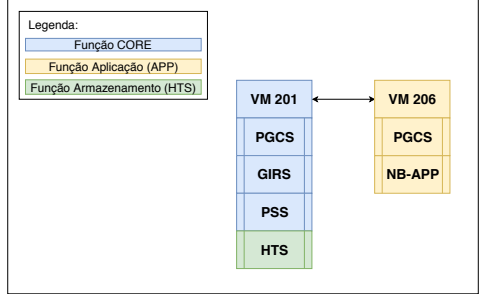

(a) 1 HTS

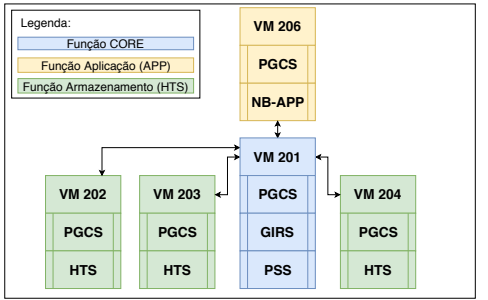

(c) 3 HTSes

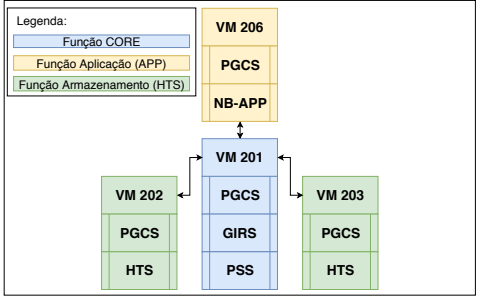

(b) 2 HTSes

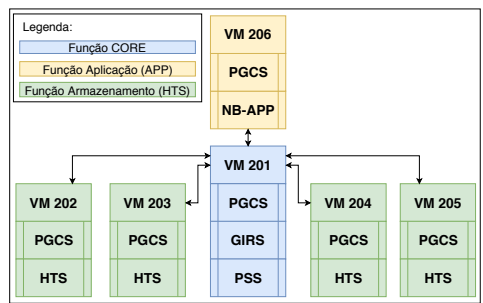

(d) 4 HTSes

Figura 2. Cenários de VMs variando de 1 a 4 HTSes.

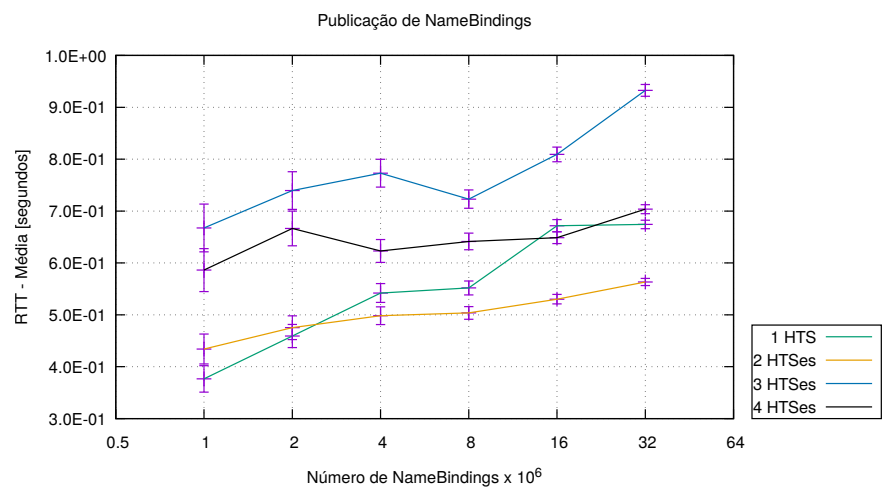

Figura 3. Tempo médio de ida e volta - Round Trip Time - (RTT) necessário para publicação de NBs variando de 1 a 4 HTSes.

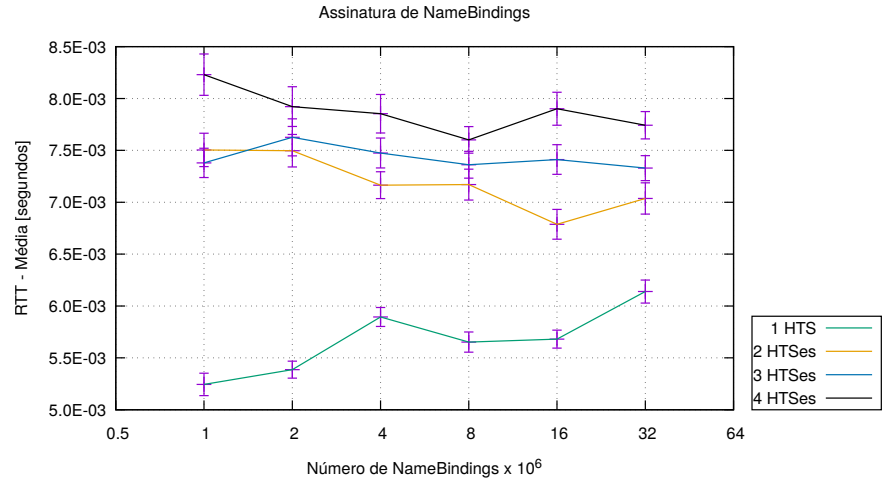

Figura 4. RTT para assinatura de NBs variando de 1 a 4 HTSes. 
tempo médio de publicação é o tempo gasto para envio e confirmação de armazenamento, pelo HTS, de mil NBs. Isso é feito através de uma única mensagem NG fragmentada e transportada sobre Ethernet. O tempo médio de assinatura pode ser comparado ao modelo de um DNS local [Alberti et al. 2017]. Ou seja, o tempo médio para solicitar um NB dentre os milhões publicados. Nas avaliações aqui realizadas, esse tempo sempre foi inferior a 8,5 milissegundos, o que indica que, de forma prática, essa solução é viável para resolver nomes em um domínio local, tratando-se de uma alternativa a outros sistemas já estabelecidos para esse fim.

\section{Considerações Finais}

A experimentação da NovaGenesis utilizando máquinas virtuais se mostrou válida por contribuir com a evolução da arquitetura e a avaliação da mesma em cenários de múltiplos nós de cache intradomínio. A alteração no número de HTSes não implica necessariamente em um grande aumento de atraso, o que mostra que a solução pode ser escalável. A utilização de um ambiente totalmente virtual se mostrou flexível às demandas de execução e coordenação dos testes. Em ambientes com computadores físicos, a execução do mesmo seria mais dispendiosa e necessitaria de um tempo maior para realização. Foi dado um passo sólido ao estabelecer um protocolo de experimentação e na obtenção de dados replicáveis. Como próximos passos será possível realizar testes em plataformas diferentes, sejam elas contêineres, computadores físicos ou testbeds de experimentação e comparálos com os resultados aqui obtidos.

\section{Agradecimentos}

Este trabalho foi parcialmente financiado pela RNP, com recursos do MCTIC, processo No 01250.075413/2018-04, sob o projeto Centro de Referência em Radiocomunicações (CRR) do Instituto Nacional de Telecomunicações - Inatel, Brasil. Os autores agradecem também a FAPEMIG, CNPq, CAPES e IFMG Campus Ouro Branco.

\section{Referências}

Alberti, A. M., Casaroli, M. A. F., Singh, D., e da Rosa Righi, R. (2017). Naming and name resolution in the future internet: Introducing the NovaGenesis approach. Future Generation Computer Systems, 67(September):163-179.

Alberti, A. M., Bontempo, M. M., Santos, J. R., Cerqueira, A. S., e da Rosa Righi, R. (2018). NovaGenesis Applied to Information-Centric, Service-Defined, Trustable IoT/WSAN Control Plane and Spectrum Management. MDPI - Sensors, PP:1-34.

Chai, W. K., Pavlou, G., Kamel, G., Katsaros, K. V., Wang, N., and Member, S. (2018). A Distributed Interdomain Control System for Information-Centric Content Delivery. IEEE Systems Journal, PP:1-12.

Conti, M., Chong, S., Fdida, S., Jia, W., Karl, H., Lin, Y.-D. D., Mähönen, P., Maier, M., Molva, R., Uhlig, S., e Zukerman, M. (2011). Research challenges towards the Future Internet. Computer Communications, 34(18):2115-2134.

Ferreira, F. A., Miranda, F. S., Rosário, C. d., D’Ávila, V. H. D., e Alberti, A. M. (2017). NovaGenesis no Ambiente FIBRE: Primeiras Impressões. pages 6-9, Belém. SBRC.

Salmito, T., Ciuffo, L., Machado, I., Abelem, A. (2014). FIBRE an international testbed for future internet experimentation. Pages 969-976. Florianópolis. SBRC 